\title{
Interstellar extinction in the direction of the Aquila Rift
}

\author{
V. Straižys ${ }^{1}$, K. Černis ${ }^{1}$, and S. Bartašiūtè $\dot{e}^{1,2}$ \\ 1 Institute of Theoretical Physics and Astronomy, Vilnius University, Goštauto 12, Vilnius 2600, Lithuania \\ e-mail: cernis@itpa.lt \\ 2 Astronomical Observatory of Vilnius University, Čiurlionio 29, Vilnius 2009, Lithuania \\ e-mail: stanislava.bartasiute@ff.vu.1t
}

Received 28 February 2003 / Accepted 14 April 2003

\begin{abstract}
The distance dependence of interstellar extinction in the direction of the Aquila Rift is investigated using 473 stars observed in the Vilnius photometric system. The front edge of the dark clouds in the area is found to be at $225 \pm 55 \mathrm{pc}$ and the thickness of the cloud system is about $80 \mathrm{pc}$. The maximum extinction $A_{V}$ in the clouds is close to 3.0 mag. Two stars with larger extinction are found and discussed. Since the new distance of the clouds is larger than the previously accepted distance, the cloud system mass should be increased to $2.7 \times 10^{5} M_{\odot}$ which is close to the virial mass estimated from the CO velocity dispersion. Additional arguments are given in favor of the genetic relation between the Serpens and the Scorpio-Ophiuchus dark clouds.
\end{abstract}

Key words. stars: fundamental parameters - ISM: dust, extinction - ISM: clouds - ISM: individual objects: Aquila Rift ISM: individual objects: Serpens molecular cloud

\section{Introduction}

Starting from Cygnus, in the direction of the Galactic center the Milky Way appears to split into two branches. The southern branch runs through Cygnus, Vulpecula, Sagitta, Aquila and Scutum, entering the Galactic central bulge in Sagittarius. The northern branch crosses Vulpecula and Aquila and disappears in the northern part of the Serpens Cauda and Ophiuchus constellations, being covered by numerous dust clouds. This complex of dark clouds usually is called the Aquila Rift.

The distances and extinction properties of these clouds are known only with low accuracy. So far the area is poorly investigated by modern photometric methods in the optical range. The collected $U B V$ and $M K$ data have been used for a crude estimate of the dependence of interstellar extinction on distance in the Rift direction (FitzGerald 1968; Neckel \& Klare 1980; Forbes 1985 and others). A sudden appearance of reddened stars at $200-250 \mathrm{pc}$ is observed. According to the summary of Dame \& Thaddeus (1985), the estimated distance of the Aquila Rift is $200 \pm 100 \mathrm{pc}$.

A field around the core of one of the densest Serpens molecular clouds (at $\left.18^{\mathrm{h}} 30^{\mathrm{m}},+1^{\circ} 14.5^{\prime}, 2000.0\right)$ with active star formation has attracted more attention (see the review article by Eiroa 1991). The distance of the cloud has been discussed by Strom et al. (1974), Chavarria et al. (1987, 1988), Zhang et al. (1988), de Lara \& Chavarria (1989), de Lara et al. (1991) and Straižys et al. (1996).

Send offprint requests to: V. Straižys, e-mail: straizys@itpa. It
Stars and other sources in the area have been well covered by the infrared surveys: by IRAS in the far infrared and by 2MASS in the JHK range. Also, numerous radioastronomical studies in the lines of $\mathrm{HI}, \mathrm{CO}, \mathrm{H}_{2} \mathrm{CO}, \mathrm{NH}_{3}$ and $\mathrm{H}_{2} \mathrm{O}$ are available in the area. The distribution of molecules, especially of CO, shows a very close resemblance to the dust distribution (see Dame \& Thaddeus 1985; Dame et al. 1987, 2001). According to $\mathrm{CO}$ radio observations, the Aquila Rift occupies a region of irregular form between $20^{\circ}$ and $40^{\circ}$ in Galactic longitude and between $-6^{\circ}$ and $+14^{\circ}$ in Galactic latitude. However, some protrusions and blobs of gas and dust extend up to $\ell=15^{\circ}$ and $b=+20^{\circ}$.

About a decade ago we started a program of investigation of the Serpens Cauda clouds belonging to the Aquila Rift by photoelectric photometry of stars in the Vilnius sevencolor photometric system (Table 1). Our first results were published by Straižys et al. (1996), hereafter Paper I. The investigation was based on photometry and photometric classification of 105 stars down to magnitude 13, located around the core of the Serpens molecular cloud mentioned above. The size of the area investigated was about 6.5 square degrees. It was found that the dust cloud in the area appears at a distance of about $260 \mathrm{pc}$.

However, the area investigated in Paper I is only a small part of the whole complex of the Aquila Rift. The newest catalog of dark clouds of Dutra \& Bica (2002) enumerates in the Rift more than 50 clouds of different sizes. It is important to know whether all these clouds are at the same distance or they form a system with a significant depth. 


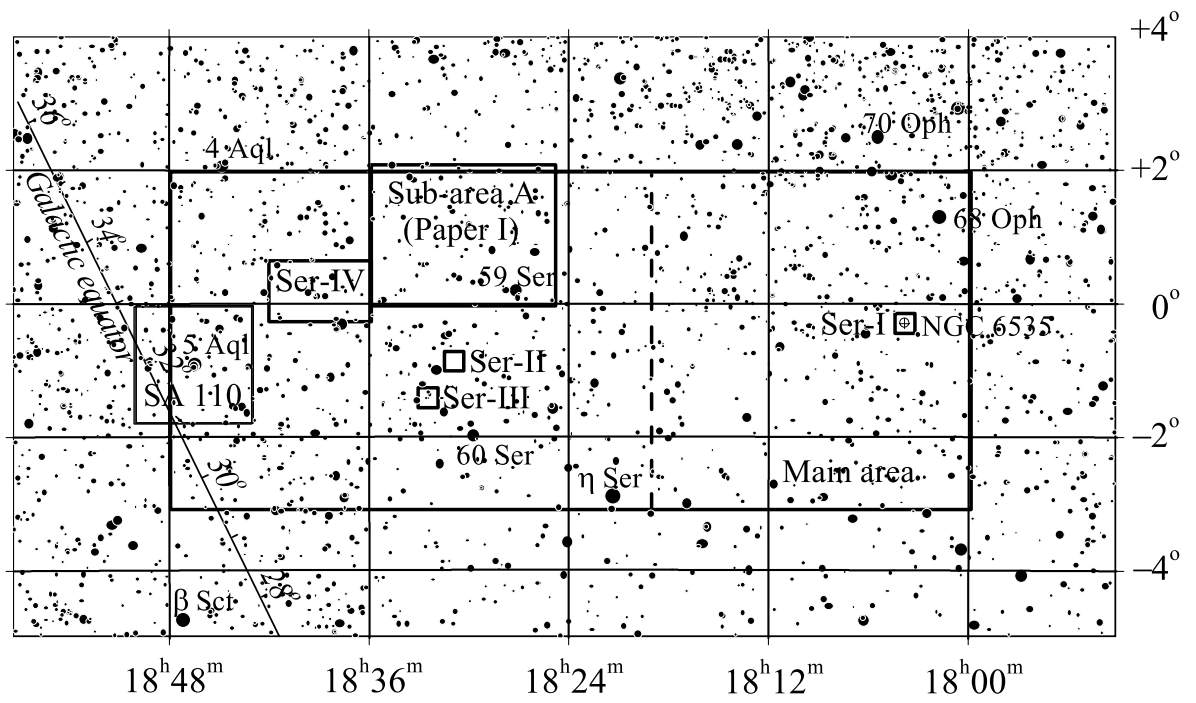

Fig. 1. The chart for the investigated Serpens Cauda area with the following sub-areas: A is the sub-area investigated in Paper I; Ser I, Ser II, Ser III and Ser IV are the sub-areas in which photoelectric standards have been measured for future CCD photometry (Černis et al. 1997 and Paper III); SA 110 is the area in which HD stars have been measured by Zdanavičius et al. (1978). The broken line at RA $=18^{\mathrm{h}} 19^{\mathrm{m}}$ divides the area into two parts with different dependence of $A_{V}$ on distance.

Table 1. Mean wavelengths and half-widths of passbands of the Vilnius photometric system.

\begin{tabular}{rrrrrrrr}
\hline \hline Passband & $U$ & $P$ & \multicolumn{1}{c}{$X$} & $Y$ & $Z$ & $V$ & \multicolumn{1}{c}{$S$} \\
\hline$\lambda(\mathrm{nm})$ & 345 & 374 & 405 & 466 & 516 & 544 & 656 \\
$\Delta \lambda(\mathrm{nm})$ & 40 & 26 & 22 & 26 & 21 & 26 & 20 \\
\hline
\end{tabular}

Table 2. Four sub-areas in which fainter stars have been measured in the Vilnius photometric system and SA 110.

\begin{tabular}{lccccrcc}
\hline \hline $\begin{array}{c}\text { Sub- } \\
\text { area }\end{array}$ & \multicolumn{2}{c}{$\begin{array}{c}\text { RA } \\
\mathrm{h}\end{array}$} & $\begin{array}{c}\text { Dec } \\
\circ\end{array}$ & $\begin{array}{r}\text { Magnitude } \\
\text { limits }\end{array}$ & $\begin{array}{c}\text { Number } \\
\text { of stars }\end{array}$ & Publ. \\
\hline Ser I & 18 & 01.3 & -00 & 18 & $11.3-14.4$ & 11 & 1 \\
Ser II & 18 & 31.5 & -00 & 50 & $11.0-13.4$ & 4 & 2 \\
Ser III & 18 & 32.5 & -01 & 23 & $10.2-12.5$ & 7 & 2 \\
Ser IV & 18 & 39.0 & +00 & 10 & $8.0-13.3$ & 45 & 2 \\
SA 110 & 18 & 46.5 & -01 & 00 & $6.9-10.7$ & 30 & 3 \\
\hline
\end{tabular}

Publications: (1) Černis et al. (1997), (2) Straižys et al. (2002c, Paper III), (3) Zdanavičius et al. (1978).

In the present paper we investigate interstellar extinction and cloud distances in a much larger area, covering $5 \times$ 10 sq. degrees. The area is limited by the following 2000.0 coordinates: RA from $18^{\mathrm{h}} 00^{\mathrm{m}}$ to $18^{\mathrm{h}} 48^{\mathrm{m}}$ and DEC from $-3.0^{\circ}$ to $+2.0^{\circ}$. Photometry in the Vilnius system was obtained in 1994, 1997 and 2001 with the $1 \mathrm{~m}$ telescope at the Maidanak Observatory in Uzbekistan. The results of photometry of 419 stars down to 11 mag and their photometric classification were published by Straižys et al. (2002b), hereafter Paper II.

Additionally, 67 fainter stars with $V$ between 10th and 14th mag were observed in four smaller sub-areas situated within the large area indicated above. These sub-areas are listed in Table 2. The measured stars in the sub-areas I-IV will be used as standards for future CCD photometry of fainter stars. Kapteyn Selected Area 110 is located at the edge of the same area. In it Vilnius photometry of 30 HD stars has been published by Zdanavičius et al. (1978). These stars were also included in the present study of interstellar extinction (see Table 2). Magnitudes and color indices of stars in the sub-areas are published in the papers listed in Table 2.

In the present study the extinction data of 80 stars from Paper I were also used. Their distances are transformed to the new distance scale corresponding to the distance modulus of Hyades $V-M_{V}=3.3$. Other stars of Paper I were rejected: some of them were found to be visual binaries and for some the classification accuracy from the photometric data was too low. They are either unresolved binaries or peculiar objects.

Consequently, we had at our disposal photometry of about 600 stars in total (14 stars are common to the catalogs of Papers I and II and 8 stars are common to the catalogs of Paper II and SA 110). However, for the investigation of extinction in the area we used 473 stars only, after the exclusion of binary, multiple and peculiar stars. The map of the investigated area with the sub-areas is shown in Fig. 1.

\section{Interstellar extinction law}

We have identified 43 stars from our list with the 2MASS survey photometry available through the Internet (Skrutskie et al. 1997). For 19 stars, covering the range of extinctions $A_{V}$ from 0.4 to $2.5 \mathrm{mag}$, we have calculated color indices $V-K$ taking $V$ from Table 1 of Paper II, and color excesses $E_{V-K}$ taking the intrinsic $(V-K)_{0}$ from Straižys (1992, Tables 22-24). Spectral types of these stars were taken from spectroscopic classification. The least squares solution gives the equation:

$E_{V-K} / E_{Y-V}=3.295+0.347(Y-V)_{0} \pm 0.464$. 
This equation shows that the ratio $E_{V-K} / E_{Y-V}$ varies from $\sim 3.4$ for A-type stars to $\sim 3.6$ for $\mathrm{K}$ giants. Since the ratio $E_{Y-V} / E_{B-V} \approx 0.8$ for $\mathrm{A}$ stars and $\approx 0.85$ for $\mathrm{K}$ giants, Eq. (1) leads to $E_{V-K} / E_{B-V}$ between 2.7 and 3.1 and $R_{B V}=$ $1.1 E_{V-K} / E_{B-V}$ between 3.0 and 3.4. These values of $R_{B V}$ are very close to the ratio given by the normal interstellar extinction law (Straižys 1992).

According to Cardelli et al. (1988, 1989), the form of the extinction law in the visible and the infrared ranges, defining the ratio $R_{B V}$, is well correlated with its form in the ultraviolet. Thus, we may accept that in the Serpens dark clouds of medium density the extinction law is normal, i.e., typical for the diffuse dust. This is expected, since the area does not contain young hot and luminous stars which may modify grain sizes in the interstellar medium. Only in the dense core of the Serpens molecular cloud, in the vicinity of some B-type stars embedded in the cloud, Chavarria et al. (1988) and de Lara et al. (1991) have found a larger than normal $R_{B V}$ ratio. However, in other parts such interaction between the hot stars and interstellar dust is not observed. Thus, in the calculation of the reddening-free $Q$-parameters we used the ratios of color excesses corresponding to the normal extinction law.

\section{Quantification of stars and their interstellar reddening}

At the beginning all known and suspected binary stars among the observed stars were identified and excluded from further investigation of interstellar extinction. For this we have identified visual binaries in the Washington Double Star Catalog (Mason et al. 2002). A number of stars were suspected as binaries by inspecting their images in Internet's virtual telescope SkyView of NASA based on the Digital Sky Survey scans of the Palomar atlas plates (http://skyview.gsfc.nasa.gov). About $8 \%$ of stars were suspected as binary or multiple stars since their images were found to be non-symmetrical. In some cases close satellite stars were detected: they also might affect photometry of the main component. The real and suspected binaries are identified in the catalogs of Papers II and III.

For the determination of spectral classes and absolute magnitudes of stars from color indices we used the interstellar reddening-free $Q$-parameters defined by the equation:

$Q_{1234}=\left(m_{1}-m_{2}\right)-\left(E_{12} / E_{34}\right)\left(m_{3}-m_{4}\right)$,

where

$E_{k, \ell}=\left(m_{k}-m_{\ell}\right)_{\text {reddened }}-\left(m_{k}-m_{\ell}\right)_{\text {intrinsic }}$.

Two independent methods described in Straižys et al. (2001b) were applied: (1) the $\sigma Q$ method which uses matching of 14 different reddening-free $Q$ parameters of a program star to those of about 8400 stars with known spectral and luminosity classes in the $M K$ system, metallicities and peculiarities and (2) the $Q, Q$ method which uses interstellar reddening-free $Q, Q$ diagrams calibrated in spectral classes and absolute magnitudes. The last method was used only for G5-K-M stars (the $Q_{U P Y}$, $Q_{X Z S}$ and $Q_{X Z S} Q_{X Y Z}$ diagrams). For spectral classes earlier than G5 only the $\sigma Q$ method was used. For each program

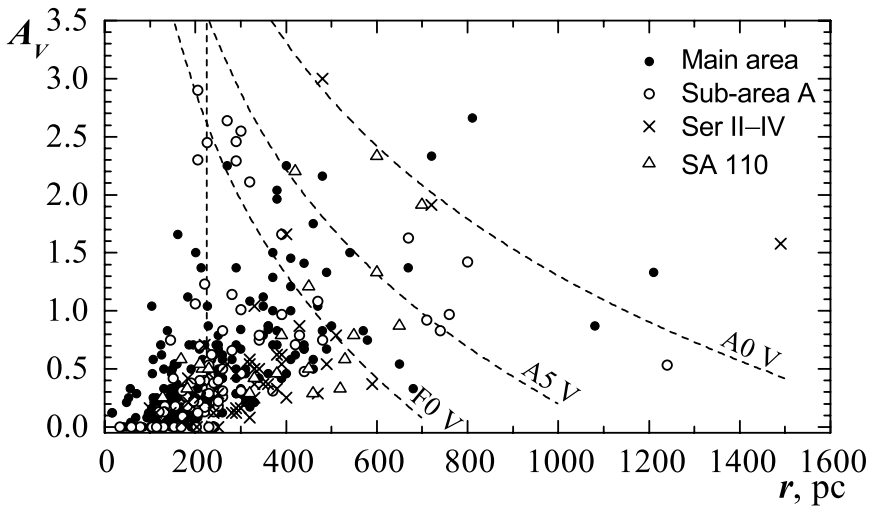

Fig. 2. Interstellar extinction $A_{V}$ plotted against distance $r$ in parsecs for the east part of the Serpens Cauda area with RA between $18^{\mathrm{h}} 19^{\mathrm{m}}$ and $18^{\mathrm{h}} 48^{\mathrm{m}}$. The vertical broken line shows the accepted distance of the front dust layer at $225 \mathrm{pc}$. The broken curves show the limiting magnitude effect for A0 V, A5 V and F0 V stars of $V=12 \mathrm{mag}$.

star three closest $M K$ stars were selected. Absolute magnitudes were taken from the $M K$ type tabulation of Straižys (1992).

The results of photometric quantification of stars and determination of their interstellar extinctions and distances are given in Paper III (Straižys et al. 2002c). For the transformation of color excesses to interstellar extinctions the normal value of the ratio $R_{Y V}=A_{V} / E_{Y-V}=4.16$ was used (Straižys et al. 1996). For the stars in the densest parts of the Serpens molecular cloud the larger values of $R_{Y V}$ were used (see Sect. 2). The distances $r$ of the stars were calculated by the equation

$\log r=\frac{V-A_{V}+5-A_{V}}{5}$.

The expected errors are: \pm 0.03 mag for $E_{Y-V}, \pm 0.1 \mathrm{mag}$ for $A_{V}$ and $\pm 25 \%$ for distance.

Papers I and III contain 38 stars closer than 250 pc with photometric distances and Hipparcos parallaxes available. Differences of the photometric and trigonometric distances of 32 stars fall within the $\pm 25 \%$ limits, as expected from the accuracy of the photometrically determined absolute magnitudes and the parallax errors. Larger differences for the remaining six stars may be explained by the photometric luminosity errors caused by undetected duplicity or peculiarity. These stars are not of decisive importance in the future study of interstellar extinction in the area. A similar comparison of photometric and trigonometric distances was done earlier in the California nebula region (Straižys et al. 2001a) and in the Aries molecular cloud region (Straižys et al. 2002a). In all cases the agreement of distance scales was found to be sufficiently good, with no systematic differences.

\section{Interstellar extinction versus distance}

The extinctions $A_{V}$ for stars in the investigated area are plotted vs. distance in Figs. 2 and 3 for two parts of the area. The division line is at RA $=18^{\mathrm{h}} 19^{\mathrm{m}}$, which separates the western part (from the Galactic equator to $b \sim 7^{\circ}$ ) with heavier extinction and the eastern part $\left(b \sim 7-11^{\circ}\right)$ with smaller extinction. On both figures the stars from the main area (Paper II) are plotted as dots, the stars from the molecular cloud area (Paper I) - 


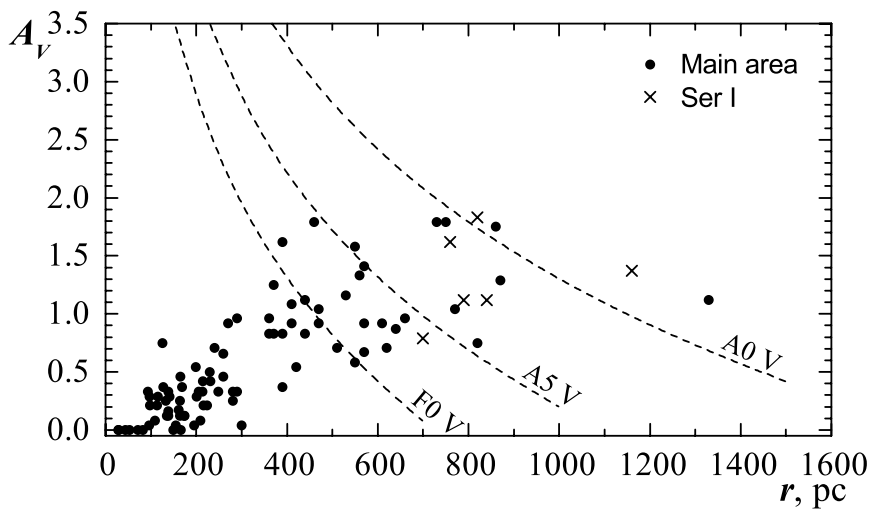

Fig. 3. Interstellar extinction $A_{V}$ plotted against distance $r$ in parsecs for the west part of the Serpens Cauda area with RA between $18^{\mathrm{h}} 00^{\mathrm{m}}$ and $18^{\mathrm{h}} 19^{\mathrm{m}}$. The limiting magnitude curves are described in Fig. 2.

as circles, the stars from Areas I to IV - as crosses, the stars from SA 110 - as triangles.

Figure 2 shows that the majority of stars in the graph form a wedge-shaped belt, which starts at the origin of the coordinates. The stars with nearly zero-reddening extend from the Solar vicinity up to $\sim 300 \mathrm{pc}$. The upper limit of the wedge grows up with the distance and at $160 \mathrm{pc} A_{V}$ reaches $\sim 0.8 \mathrm{mag}$. Only one star, BD-2 4634 (or No. 31 in Paper II) shows an anomalous value of extinction: at $104 \mathrm{pc} A_{V}$ is 1.04 . It is not excluded that the star may be an unresolved binary. At $160 \mathrm{pc}$ a sudden increase of $A_{V}$ takes place. The majority of stars continue to follow the wedge-shaped pattern, however, a smaller number of stars exhibit much larger values of $A_{V}$ : up to 1-3 mag and even more. This is an indication that somewhere between 200 and $400 \mathrm{pc}$ a network of dense dust clouds starts to appear. However, these clouds do not cover all the area since many stars at these distances show only moderate extinction, between 0.4 and $1.0 \mathrm{mag}$.

Let us estimate the distance of the nearest dust concentrations. The standard deviation of distances is expected to be about $\pm 0.25 r$. This means that the apparently closest heavily reddened stars may appear at a distance of $r-0.25 r=160 \mathrm{pc}$, i.e., $r=160 / 0.75=213$ pc. Another estimation of the front edge distance of the clouds comes from the unreddened or slightly reddened stars, exhibiting the largest apparent distances. For them $r+0.25 r=300$ pc, i.e., $r=300 / 1.25=$ $240 \mathrm{pc}$. Thus, the real distance of the front dark cloud, responsible for the rise of extinction at $r>160 \mathrm{pc}$, is between 213 and $240 \mathrm{pc}$, i.e. approximately at $225 \pm 56 \mathrm{pc}$. It is interesting that the stars, exhibiting heavy extinction within this distance range do not show any correlation with the apparent richness of the background stars.

In Fig. 3, which shows the stars at greater distances from the Galactic plane, the extinction up to $100 \mathrm{pc}$ is nearly zero. Beyond this distance, the extinction increases gradually up to $300-400 \mathrm{pc}$ and the maximum $A_{V}$ values do not exceed $1.8 \mathrm{mag}$. This means that the extinction in the area is much lower and more uniform in comparison with lower Galactic latitudes.

There is no possibility of estimating the largest extinction value at different distances, since in Figs. 2 and 3 a strong selection effect is present. The dotted lines show the limiting magnitude effect at $V=12$ mag for the stars of spectral classes A0 V, A5 V and F0 V. The stars of these spectral types above the corresponding curves are accessible only if the $V$ magnitude limit is set at $V>12$. The limiting magnitude in various directions of the area varies from 10 to 13 . Consequently, the distribution of stars in the figures is strongly affected by selection, and the number of heavily reddened stars at distances $>400 \mathrm{pc}$ is considerably reduced. Therefore, we are not able to give any maximum or medium extinction values at these distances. Moreover, a number of more distant dust clouds may be present. However, the lowest extinction value in the area can be estimated with sufficiently high accuracy. At distances $r>400 \mathrm{pc}$ it is about $0.5 \mathrm{mag}$. The stars with such low extinction are seen through windows between the dust clouds.

The star BD $-013542(V=9.24)$ deserves a special discussion. According to Hiltner \& Iriarte (1955) its spectral type is B8 Ia:. Nassau \& Stephenson (1963) in their Catalog of Luminous Stars in the Northern Milky Way list this star as LS IV -01 4 with spectral type $\mathrm{OB}+\mathrm{R}$ where $\mathrm{R}$ means "reddened". Bidelman (1988) finds in its spectrum a "very weak broad $\mathrm{H} \alpha$ emission". Our photometric classification gives a spectral class B2/3 and a luminosity II-III (uncertain). If the star were B3 II-III, its $A_{V}$ should be 5.0, $M_{V} \approx-4.0$ and the distance $440 \mathrm{pc}$. It is unlikely that so heavily reddened star is at this small distance and in the area with a relatively rich Milky Way background. If we accept the B8 Ia type, its $A_{V}$ becomes $4.6 \mathrm{mag}, M_{V} \approx-7.3 \mathrm{mag}$ and the distance $2.4 \mathrm{kpc}$. This combination of $A_{V}$ and $r$ seems to be more acceptable, since the star is only at $+1^{\circ}$ latitude. The star deserves careful spectral and photometric investigation.

Another interesting star from our list is BD -024676 , with $V=10.25$. This is irregular Lb-type variable CZ Ser of spectral class M6 III (photometric classification). The same spectral class is given by Hanson \& Blanco (1975) from low-dispersion objective-prism spectra. The General Catalogue of Variable Stars (Kholopov 1987) and Sloan \& Price (1998) list a spectral class of M6.5 for the star. The star is an infrared source IRAS 18347-0241 and it has a circumstellar silicate and CO envelope (Sloan \& Price 1998; Kerschbaum \& Olofsson 1999). We obtain its reddening $E_{Y-V} \approx 0.84$. If all this reddening is of interstellar origin, its $A_{V}=3.5 \mathrm{mag}$, and with $M_{V}=0.0 \mathrm{mag}$ a distance of $225 \mathrm{pc}$ follows. However, since part of the reddening is circumstellar, its $A_{V}$ and distance are very uncertain (see also a discussion of Olofsson et al. 2002).

\section{Discussion and conclusions}

The investigations described in Paper I and the present paper give evidence that the dust clouds of the Aquila Rift begin at 200-250 pc distance. These clouds cover the whole investigated area including regions with different richness of the background stars, seen on the red Palomar Sky Survey plates. This means that the ornament of dark lanes in the area is formed by more distant dust concentrations. These dark features are observable in the Rift up to Galactic latitude of $\sim 5^{\circ}$. At higher 
latitudes distant stars of the Milky Way probably are attenuated by the $200-250$ pc clouds only.

There is no direct way to determine how deep the system of the Aquila Rift clouds is. As follows from the apparent obscuration of the Milky Way and from the low-velocity CO distribution, the Aquila Rift extends by about $20^{\circ}$ both in Galactic longitude and latitude. At 225 pc distance this corresponds to $82 \mathrm{pc}$. This value may characterize the cloud system depth if it is more or less spherical. This diameter of the cloud system fits perfectly the equation between the logarithms of the CO line widths and the logarithms of the cloud radii derived by Dame et al. $(1985,1986)$. Consequently, if the front edge of the cloud system is at $225 \mathrm{pc}$ and the far edge at $310 \mathrm{pc}$, the center distance is at about $270 \mathrm{pc}$. At this distance from the Sun the maximal distance of the clouds from the Galactic plane is $70 \mathrm{pc}\left(\sim 15^{\circ}\right)$.

At about $15^{\circ}$ longitude the northern part of the Milky Way (or the Central Bulge) reappears again, however it is mottled by numerous dark lanes which are very similar to the lanes in Serpens Cauda. Probably these dust concentrations belong to the same cloud system but they are nearer to the center or the far edge (up to $310 \mathrm{pc}$ from the Sun).

On the other hand, the Ophiuchus-Scorpio complex of dust and molecular clouds between longitudes $350-0^{\circ}$ extends from $-7^{\circ}$ to $+22^{\circ}$ in latitude. At a distance of the front edge of the clouds of 150 pc (Straižys 1984, after transforming to the Hyades distance modulus of $3.3 \mathrm{mag}$ ) the diameter of the clouds is $\sim 80 \mathrm{pc}$, and the center of the cloud system is at a distance of $190 \mathrm{pc}$. At this heliocentric distance the height above the Galactic plane of $22^{\circ}$ corresponds to $\sim 80 \mathrm{pc}$. Thus, both the Serpens clouds and the Ophiuchus-Scorpio clouds reach about the same distance above the plane. This is an argument for a common origin of both the Aquila Rift and the OphiuchusScorpio clouds. A connection of both systems of molecular clouds has been suspected by Lebrun \& Huang (1984) and Dame \& Thaddeus (1985) on the ground of the surface distribution and radial velocities of $\mathrm{CO}$ radio emission. They have also related this cloud system to the clouds in Lupus, on the other side of the Galactic center. Dame et al. (1987) note that all these local molecular clouds can be readily distinguished from the more distant ones by their low velocity (less than $20 \mathrm{~km} \mathrm{~s}^{-1}$ ) and a wide extent to the northern latitudes.

According to Dame et al. (1987) the mass of the Aquila Rift clouds, estimated from the intensities of CO lines, is about $1.5 \times 10^{5} M_{\odot}$ at a distance of $200 \mathrm{pc}$. However, the center of the cloud system is probably at about 270 pc, i.e., 1.35 times farther. The new distance leads to the increase of the surface density of gas by a factor of 1.8. Thus the total mass of the Aquila Rift clouds may be as large as $2.7 \times 10^{5} M_{\odot}$ which is still lower than the virial mass of the clouds calculated by Dame \& Thaddeus (1985) from the radial velocity dispersion (or CO line widths) and transformed to the new distance and hence larger cloud radius.

The above conclusions about the distribution of the molecular and dust clouds receive support from the existing Milky Way surveys in the far infrared wavelengths. A glance at the 240, 100 and $50 \mu \mathrm{m}$ dust emission maps obtained by IRAS and COBE/DIRBE (http://skyview.gsfc.nasa.gov; Schlegel et al. 1998) shows that the investigated area up to $b=+5^{\circ}$ emits a strong and uniform radiation with some intensity peaks. The strongest source of the far IR radiation is the complex W40 near the star 60 Ser, containing a molecular cloud, a H II region and a number of point IR sources. This complex is considered to be unrelated with the Aquila Rift, being at a distance of 400700 pc (Smith et al. 1985; Vallee \& MacLeod 1994; Shuping et al. 1999). Its distance is very uncertain, being determined mostly by the kinematical method, combining radial velocities from molecular radio lines and the rotation curve of the Galaxy. Reyle \& Robin (2002) using the Point Source Catalogue of the DENIS infrared survey have identified a young open cluster in the complex. The extinction $A_{V}$ in front of the complex is estimated by different authors to be from 9 to $17 \mathrm{mag}$. Since the Galactic latitude of the complex is $+3.5^{\circ}$ only, this extinction originates not only in the Aquila Rift clouds.

Another somewhat fainter far IR source is the core of the Serpens molecular cloud investigated in our Paper I. At higher Galactic latitudes the dust and $\mathrm{CO}$ emission drops out and almost vanishes at $b \geq 15^{\circ}$. Optically, both $\mathrm{W} 40$ and the Serpens molecular cloud are seen projected on a very dark foreground created by the dust lanes of the Aquila Rift.

The main conclusions of the investigation may be summarized in the following items: (1) the front edge of the dark clouds in the Aquila Rift is situated at the $225 \pm 55$ pc distance, (2) the cloud complex is about $80 \mathrm{pc}$ deep, (3) the maximum extinction in $V$ in the cloud system is close to $3.0 \mathrm{mag}$, (4) the objects at low Galactic latitudes (like the star BD 013542 and the cloud complex W40) exhibit much larger extinction and are situated far behind the Rift, (5) the Aquila Rift clouds are similar to the Ophiuchus-Scorpio clouds: both complexes reach more or less the same maximum height above the Galactic plane.

Acknowledgements. We are grateful to A. G. Davis Philip and the anonymous referee for important corrections.

\section{References}

Bidelman, W. P. 1988, PASP, 100, 1084

Cardelli, J. A., Clayton, G. C., \& Mathis, J. S. 1988, ApJ, 329, L33

Cardelli, J. A., Clayton, G. C., \& Mathis, J. S. 1989, ApJ, 345, 245

Černis, K., Bartašiūtè, S., Straižys, V., \& Janulis, R. 1997, Baltic Astron., 6, 573

Chavarria, K. C., Ocegueda, J., de Lara, E., Finkenzeller, U., \& Mendoza, E. E. 1987, in Circumstellar Matter, ed. I. Appenzeller, \& C. Jordan (Dordrecht: Reidel Publishing Company), IAU Symp., 122, 97

Chavarria, K. C., de Lara, E., Finkenzeller, U., Mendoza, E. E., \& Ocegueda, J. 1988, A\&A, 197, 151

Dame, T. M., \& Thaddeus, P. 1985, ApJ, 297, 751

Dame, T. M., Elmegreen, B. G., Cohen, R. S., \& Thaddeus, P. 1986, ApJ, 305, 892

Dame, T. M., Ungerechts, H., Cohen, R. S., et al. 1987, ApJ, 322, 706

Dame, T. M., Hartmann, D., \& Thaddeus, P. 2001, ApJ, 547, 792

de Lara, E., \& Chavarria, K. C. 1989, Rev. Mex. Astron. Astrofis., 18, 180 
de Lara, E., Chavarria, K. C., \& Lopez-Molina, G. 1991, A\&A, 243, 139

Dutra, C. M., \& Bica, E. 2002, A\&A, 383, 631

Eiroa, C. 1991, in Low Mass Star Formation in Southern Molecular Clouds, ed. B. Reipurth, ESO Scientific Report, 11, 197

FitzGerald, M. P. 1968, AJ, 73, 983

Forbes, D. 1985, AJ, 90, 301

Hanson, O. L., \& Blanco, V. M. 1975, AJ, 80, 1011

Hiltner, W. A., \& Iriarte, B. 1955, ApJ, 122, 185

Kerschbaum, F., \& Olofsson, H. 1999, A\&AS, 138, 299

Kholopov, P. N. (ed.) 1987, General Catalogue of Variable Stars, vol. III (Moscow: Nauka Publishers)

Lebrun, F., \& Huang, Y.-L. 1984, ApJ, 281, 634

Mason, B. D., Wycoff, G. L., \& Hartkopf, W. I. 2002, The Washington Double Star Catalog (http://ad.usno.navy.mil/wds/wdstext.html)

Nassau, J. J., \& Stephenson, C. B. 1963, Luminous Stars in the Northern Milky Way, vol. IV (Hamburg-Bergedorf)

Neckel, Th., \& Klare, G. 1980, A\&AS, 42, 251

Olofsson, H., Gonzalez Delgado, D., Kerschbaum, F., \& Schöier, F. L. 2002, A\&A, 391, 1053

Reyle, C., \& Robin, A. C. 2002, A\&A, 384, 403

Schlegel, D. J., Finkbeiner, D. P., \& Davis, M. 1998, ApJ, 500, 525

Shuping, R. Y., Snow, T. P., Crutcher, R., \& Lutz, B. L. 1999, ApJ, 520,149
Skrutskie, M. F., Schneider, S. E., Stiening, R., et al. 1997, in The Impact of Large Scale Near-IR Sky Surveys, ed. F. Garzon et al. (Dordrecht: Kluwer Publishing Company), 25

Sloan, G. C., \& Price, S. D. 1998, ApJS, 119, 141

Smith, J., Bentley, A., Castelaz, M., et al. 1985, ApJ, 291, 571

Straižys, V. 1984, Bull. Vilnius Obs., 67, 3

Straižys, V. 1992, Multicolor Stellar Photometry, (Tucson: Pachart Publishing House)

Straižys, V., Černis, K., \& Bartašiūtè, S. 1996, Baltic Astron., 5, 125 (Paper I)

Straižys, V., Černis, K., \& Bartašiūtè, S. 2001a, Baltic Astron., 10, 319

Straižys, V., Černis, K., \& Bartašiūtè, S. 2001b, A\&A, 374, 288

Straižys, V., Černis, K., Kazlauskas, A., \& Laugalys, V. 2002a, Baltic Astron., 11, 231

Straižys, V., Černis, K., \& Bartašiūtè, S. 2002b, Baltic Astron., 11, 321 (Paper II)

Straižys, V., Bartašiūtė, S., \& Černis, K. 2002c, Baltic Astron., 11, 417 (Paper III)

Strom, S. E., Grasdalen, G. L., \& Strom, K. M. 1974, ApJ, 191, 111

Vallee, J. P., \& MacLeod, J. M. 1994, AJ, 108, 998

Zdanavičius, K., Gurklytè, A., Sūdžius, J., Jasevičius, V., \& Kazlauskas, A. 1978, Bull. Vilnius Obs., 49, 3

Zhang, C. Y., Laurejs, R. J., Clark, F. O., \& Wesselius, P. R. 1988, A\&A, 199, 170 